\title{
The influence of simultaneous lateral grafting on clinical outcomes following one-stage implant placement: a cross- sectional analysis
}

Ausra Ramanauskaite ${ }^{1 *}$, Frank Schwarz ${ }^{2}$, Amira Begic ${ }^{2}$, Puria Parvini ${ }^{2}$, Maria Elisa Galarraga-Vinueza ${ }^{3}$ and Karina Obreja ${ }^{4}$

\begin{abstract}
Background: To investigate the influence of lateral bone augmentation procedures performed simultaneously with implant placement on peri-implant health or disease.

Material and methods: A total of 232 patients showing the same type of a two-piece implant placed either simultaneously with lateral bone grafting using a bovine bone mineral and a native collagen membrane $(n=291$ implants; test group) or at pristine bone sites without lateral bone grafting ( $n=283$ implants; control group) were enrolled in this cross-sectional analysis. Clinical outcomes (i.e., modified plaque index ( $\mathrm{mPl}$ ), bleeding on probing $(B O P)$, probing depth (PD), and mucosal recession (MR)), and the frequency of peri-implant disease were evaluated after a mean follow-up period of $9.97 \pm 6.55$ years.

Results: No differences were found between the patients in the test and control groups for any of the parameters investigated (i.e., $\mathrm{mPI}, \mathrm{BOP}, \mathrm{PD}$, and MR). For the implants in both groups, PD values of 4-6 mm were more frequently noted in the upper jaw. A significant correlation between increased PD values and a larger implant diameter was noted for test implant sites. A KM of $<2 \mathrm{~mm}$ was associated with increased MR values in both groups. The prevalence of peri-implant mucositis and peri-implantitis was $68 \%$ and $5 \%$ for the patients in the test group and $61 \%$ and $10 \%$ in the control group, respectively.
\end{abstract}

Conclusions: Simultaneous lateral grafting was associated with peri-implant tissue health and stability.

Keywords: Alveolar ridge augmentation, Guided bone regeneration, Dental implant

\section{Introduction}

It is well documented that tooth extraction is followed by inevitable dimensional changes of the alveolar ridge, leading to reduced overall ridge volume and changes in the ridge shape [1]. As a consequence, once a dental implant-supported reconstruction-is chosen as a treatment option to fill the edentulous space, bone

\footnotetext{
* Correspondence: ramanauskaite@med.uni-frankfurt.de

'Department of Oral Surgery and Implantology, Carolinum, Goethe University, Theodor-Stern-Kai 7, Building 29, 60596 Frankfurt am Main, Germany

Full list of author information is available at the end of the article
}

augmentation procedures are frequently required, either concomitant with implant placement or as a staged intervention.

In clinical situations involving horizontal alveolar ridge deficiencies, lateral bone augmentation procedures have been shown to effectively increase bone width, rendering implant placement in a second-stage surgery feasible [2]. Furthermore, procedures to regenerate the lateral alveolar ridge, when performed simultaneously with dental implant placement, have demonstrated a capacity to markedly reduce peri-implant bone defects, leading to a 
mean defect fill of $81.3 \%$ [3]. In fact, the optimal reduction of the defect height was more favorable when a combination of a grafting material and a barrier membrane was used, compared to a membrane or grafting material alone or the absence of treatment [3].

The potential influence of residual defects following lateral bone grafting has become a matter of concern, since it may be associated with an increased risk for peri-implant mucosal inflammation and subsequently progressive bone loss [4,5]. Even though the results from a recent systematic review and meta-analysis have indicated that the changes in BOP over time were minimal $[n=10$ studies; $\mathrm{WMD}=-10.02 \%$; 95\% CI $(-22.23,2.21)]$, it was also emphasized that the underlying evidence is weak. In fact, clinical and radiographic parameters to differentiate peri-implant health from disease have been inconsistently used in the evaluated studies and did not consider currently established case definitions for peri-implant disease [5].

Therefore, the aim of the present cross-sectional study was to further investigate the influence of lateral bone augmentation procedures performed simultaneously with implant placement on the maintenance of periimplant health.

\section{Materials and methods \\ Study design}

The present cross-sectional analysis included 232 partially or fully edentulous patients (134 females and 98 males) exhibiting 574 implants (Ankylos ${ }^{\circ}$, DENTSPLY Implants Manufacturing GmbH, Mannheim, Germany). All implants were placed in the Department of Oral Surgery and Implantology, Goethe University, Frankfurt, following a standardized treatment protocol. Each patient had received a detailed description of the procedure, and an informed consent form was obtained prior to participation. The procedures in this study were in accordance with the Declaration of Helsinki, as revised in 2013, and the study protocol was approved by the local ethics committee (registration number 78/ 18).

\section{Patient selection criteria}

The following inclusion criteria were applied for patient selection:

- Patients with $>18$ years of age rehabilitated with at least one Ankylos implant;

- Patients with treated chronic periodontitis and regular maintenance care;

- Non-smokers, smokers, and former smokers;

- A good level of oral hygiene as evidenced by a plaque index (PI) $<1$ at the implant level;
- Attendance of a yearly routine implant maintenance appointment.

Patients were excluded for the following conditions: systemic diseases that could influence the outcome of the therapy, such as diabetes $(\mathrm{HbA} 1 \mathrm{c}<7)$, osteoporosis; a history of malignancy, radiotherapy, chemotherapy, immunodeficiency, or antiresorptive therapy; and pregnancy or lactation at the last follow-up.

\section{Treatment protocol}

Two-piece platform-switched implants (Ankylos ${ }^{\circ}$, Dentsply Implants Manufacturing $\mathrm{GmbH}$, Mannheim, Germany) were placed in a prosthetically ideal position and, according to the manufacturer's surgical protocol, considering a subcrestal positioning of the implant shoulder. Implants in the control group displayed an intact vestibular alveolar bone wall without the need for a lateral bone grafting procedure. Implants in the test group exhibited dehiscence-type defects at the vestibular aspect, which were simultaneously filled with a particulated bovine bone mineral (Bio-Oss spongiosa granules sized $0.25-1 \mathrm{~mm}$, Geistlich, Wolhusen, Switzerland) and covered by a native collagen membrane (Bio-Gide, Geistlich, Wolhusen, Switzerland). While control sites were left to heal in a transmucosal position, all test sites were submerged for a healing period of 4 to 6 months. The implants in both test and control groups were mainly restored with fixed cemented (control 273; test 264) crowns (control 188; test 190) and bridges (control 68; test 59). Screw-retained (control 9; test 25), telescopic (control 5; test 4), and removable restorations (control 0; test 22) were less frequent.

\section{Implant and implant-site characteristics}

The following study variables were assessed for both test and control implants:

(1) Implant age (i.e., defined as time after implant placement), (2) implant location (i.e., upper or lower jaw, anterior (i.e., canine to canine) or posterior (i.e., premolar and molar region) segments), and (3) implant diameter.

\section{Clinical measurements}

The following clinical parameters were registered at each implant site using a conventional periodontal probe:

(1) Modified plaque index (mPI) (Löe et al.) [6], (2) bleeding on probing (BOP)-measured as presence/absence, (3) probing depth (PD)-measured from the mucosal margin to the probable pocket, (4) mucosal recession (MR)-measured from the restoration margin to the mucosal margin, and (5) keratinized mucosa (KM) (mm). 
Table 1 Patient and implant site characteristics

\begin{tabular}{lll}
\hline & $\begin{array}{l}\text { Control group } \\
(n=283 \text { implants })\end{array}$ & $\begin{array}{l}\text { Test group } \\
(n=291 \text { implants })\end{array}$ \\
\hline Patient number & $n=121$ & $n=111$ \\
Patient age & $65.19 \pm 12.2$ years & $64.84 \pm 12.15$ years \\
Patient gender (female/male) & $70 / 51$ & $64 / 47$ \\
Implant age years (mean \pm SD) & $11.76 \pm 7.19$ years & $8.12 \pm 5.21$ years \\
Location upper jaw: anterior/posterior segment $(n)$ & $47 / 158$ & $35 / 79$ \\
Location lower jaw: anterior/posterior segment $(n)$ & $16 / 62$ & $48 / 129$ \\
Keratinized mucosa $(\mathrm{mm})$ & $2.84 \pm 1.61$ & $2.47 \pm 1.52$ \\
Implant diameter: $3.5 / 4.5 / 5.5 \mathrm{~mm}(n)$ & $233 / 48 / 2$ & $225 / 61 / 5$ \\
\hline
\end{tabular}

Modified PI, BOP, PD, and MR measurements were performed at six sites per implant: mesiobuccal (mb), midbuccal (b), distobuccal (db), mesiooral (mo), midoral (o), and distooral (do). KM measurement was performed at three aspects per implant: mesiobuccal (mb), midbuccal (b), and distobuccal (db).

The presence of peri-implant disease at each implant site was assessed according to established case definitions [7]:

- Peri-implant mucositis defined as the presence of BOP and/or suppuration with on gentle probing with or without increased PDs compared to previous examinations, and an absence of bone loss beyond crestal bone level changes resulting from initial bone remodeling.

- Peri-implantitis defined as the presence of BOP and/ or suppuration on gentle probing, increased PDs compared to previous examination, and the presence of bone loss beyond crestal bone level changes resulting from initial bone remodeling.

\section{Radiographic assessment}

When clinical signs suggested the presence of periimplant tissue inflammation, panoramic radiographs were assessed. To evaluate the bone-level changes at the implant sites, the obtained radiographs were compared with the baseline situation (i.e., radiographs taken following the placement of the final prosthetic reconstruction). After digitalization of the radiographs (Microtek ScanMaker i800 Plus, Hsinchu, Taiwan; LaserSoft Imaging AG, Kiel, Germany), measurements (i.e., bonelevel changes between the baseline and follow-up radiographs) were performed employing the Sidexis XG software (Sirona Dental Systems GmbH, Bensheim, Germany). The measurement scale was based on the known implant height. Two reference horizontal lines were used: one marked the most coronal point of the peri-implant bone crest at mesial and distal sites (BC), and another traced the implant's most apical point (AP). Vertical lines parallel to the reference line crossing the long axis of the implant were traced perpendicularly to the $\mathrm{BC}$ and AP at mesial and distal sites.

Table 2 Clinical parameters (mean \pm SD, median, and 95\% Cl)

\begin{tabular}{|c|c|c|c|c|c|c|c|}
\hline Clinical parameters & $\begin{array}{l}\text { Control group } \\
\text { (mean } \pm \text { SD) }\end{array}$ & Median & $95 \% \mathrm{Cl}$ & $\begin{array}{l}\text { Test group } \\
(\text { mean } \pm \text { SD) }\end{array}$ & Median & $95 \% \mathrm{Cl}$ & $p$ \\
\hline \multicolumn{8}{|c|}{ Modified plaque index } \\
\hline Patient-level & $0.46 \pm 0.43$ & 0.33 & $0.38-0.54$ & $0.49 \pm 0.42$ & 0.50 & $0.41-0.57$ & 0.56 \\
\hline Implant-level & $0.49 \pm 0.41$ & 0.33 & $0.44-0.54$ & $0.51 \pm 0.43$ & 0.50 & $0.46-0.56$ & 0.51 \\
\hline \multicolumn{8}{|c|}{ Bleeding on probing (\%) } \\
\hline Patient-level & $33.07 \pm 30.37$ & 33 & $27.36-38.79$ & $32.26 \pm 30.88$ & 17 & $26.71-37.82$ & 0.084 \\
\hline Implant-level & $36.77 \pm 32.23$ & 33 & $33-40.10$ & $35.53 \pm 32.67$ & 33 & $31.76-39.30$ & 0.648 \\
\hline \multicolumn{8}{|c|}{ Probing depth (mm) } \\
\hline Patient-level & $3.0 \pm 0.72$ & 2.83 & $2.86-3.13$ & $2.86 \pm 0.96$ & 2.67 & $2.68-3.03$ & 0.22 \\
\hline Implant-level & $3.0 \pm 0.85$ & 2.83 & $2.90-3.11$ & $2.78 \pm 0.86$ & 2.67 & $2.68-2.88$ & 0.002 \\
\hline \multicolumn{8}{|c|}{ Mucosal recession (mm) } \\
\hline Patient-level & $0.17 \pm 0.34$ & 0 & $0.10-0.23$ & $0.18 \pm 0.36$ & 0 & $0.11-0.24$ & 0.79 \\
\hline Implant-level & $0.16 \pm 0.36$ & 0 & $0.12-0.20$ & $0.19 \pm 0.36$ & 0 & $0.16-0.24$ & 0.221 \\
\hline
\end{tabular}


Table 3 Prevalence of peri-implant health and disease

\begin{tabular}{lllll}
\hline & Patient-level control group & Test group & Implant-level control group & Test group \\
\hline Healthy & 32 & 33 & 79 & 83 \\
Peri-implant mucositis & 68 & 82 & 181 & 192 \\
Peri-implantitis & 11 & 6 & 23 & 16 \\
\hline
\end{tabular}

\section{Statistical analysis}

The statistical analysis was performed using a commercially available software program (SPSSStatistics 26.0: IBM Corp., Ehningen, Germany). Descriptive statistics (means, standard deviations, medians, and 95\% confidence intervals) were calculated for $\mathrm{MPI}, \mathrm{BOP}, \mathrm{PD}$, and MR values. The analysis was performed at the patient and implant levels. The data were tested for normality by means of the Shapiro-Wilk test. Between-group comparisons of clinical outcomes were accomplished using the unpaired $t$ test. Logistic regression analyses based on the implant-level data were used to depict relationships between $\mathrm{BOP}, \mathrm{PD}$, or MR values and the following variables: implant location (anterior/posterior), diameter $(<$ $4.5 / \geq 4.5 \mathrm{~mm})$, and $\mathrm{KM}(<2 / \geq 2 \mathrm{~mm})$. The alpha error was set at 0.05 .

\section{Results}

\section{Patient, implant, and implant site characteristics}

The test group enrolled 64 women and 47 men with a total of 283 implants. The sample in the control group consisted of 70 women and 51 men with 291 implants. The mean estimated patient age in the test and control groups was $64.84 \pm 12.15$ and $65.19 \pm 12.2$ years, respectively.

The mean implant functioning time was $8.12 \pm 5.12$ years for the test group and $11.76 \pm 7.19$ years for the control group. Majority of the included implants had a diameter of $3.5 \mathrm{~mm}$ (test $77 \%$; control $82 \%$ ) with a mean facial KM width of $2.47 \mathrm{~mm}$ in the test group and of $2.84 \mathrm{~mm}$ in the control group (Table 1). In the test group, most implants were located in the posterior region of the lower jaw (44\%), whereas the most common implant location in the control group was the posterior segment of the upper jaw (56\%).

\section{Clinical measurements}

The clinical measurements are presented in Table 2. In general, all patients and implant sites exhibited low mPI scores (patient-level: test 0.49, control 0.46; $p=0.56$ : implant-level: test 0.51 and 0.49 , respectively; $p=0.51$ ). Mean BOP scores and MR values were comparable between the patients in the test and control groups (test $32.26 \%$ and $0.18 \mathrm{~mm}$; control $33.07 \%$ and $0.17 \mathrm{~mm}$, respectively), and similar at test and control implant sites (test $35.53 \%, \quad 0.19 \mathrm{~mm}$; control $36.77 \%, 0.16 \mathrm{~mm}$, respectively). With regards to the mean PD scores, based on the patient-level data, no difference was noted between the two groups (test $2.86 \mathrm{~mm}$ : control $3.0 \mathrm{~mm} ; p$ $=0.22$ ), whereas implant-level estimations pointed toward higher mean PD values at the test implant sites (3.0 mm vs. $2.78 \mathrm{~mm} ; p=0.002$ ).

\section{Incidence of peri-implant disease}

The frequency distribution of peri-implant disease in the test and control groups is summarized in Table 3. According to the given case definitions, $68 \%$ of the patients in the test group and $61 \%$ of the patients in the control group were diagnosed with peri-implant mucositis, while peri-implantitis was diagnosed in $5 \%$ of the patients in the test and in $10 \%$ of the patients in the control group. At the implant level, the corresponding values amounted to $66 \%$ and $5 \%$ in the test group and $64 \%$ and $8 \%$ in the control group, respectively (Table 3 ).

\section{Regression analysis}

Cross-tabulations between selected independent variables (PD, MR, and BOP values) and local factors (i.e., implant region, implant diameter, and $\mathrm{KM}$ ) in the test and control groups are summarized in Tables 4 and 5 .

In both groups, implants located in the upper jaws were more frequently associated with PD values of 4-6 $\mathrm{mm}$ than in the lower jaws ( $p=0.001$, respectively). In the test group, PD values were commonly higher at implants exhibiting larger diameters (i.e., 4.5 and $5.5 \mathrm{~mm}$ vs. $3.5 \mathrm{~mm} ; p=0.04$ ). For control group implants, BOP scores of $>67 \%$ and PD values of $4-6 \mathrm{~mm}$ were more frequently noted at implant sites exhibiting $\mathrm{KM}<2 \mathrm{~mm}$ ( $p=0.03$ and $p=0.017$, respectively). Moreover, at both test and control implants, KM values of $<2 \mathrm{~mm}$ were correlated with increases in MR valuers $(p=0.001$, respectively).

\section{Discussion}

The present cross-sectional study aimed at evaluating the influence of lateral bone augmentation procedures performed simultaneously with implant placement on peri-implant health or disease.

Based on the present data, none of the investigated clinical parameters (i.e., mPI, BOP, PD, and MR) differed between the patients treated with simultaneous lateral bone augmentation and those treated with implants 
Table 4 Test group ( $n=291$ implants) cross-tabulations: a) BOP/PD/MR values and (1) implant region, (2) implant diameter, and (3) KM

\begin{tabular}{|c|c|c|c|}
\hline \multirow[t]{2}{*}{ (1) BOP values } & \multicolumn{3}{|c|}{ Implant region } \\
\hline & Upper jaw & \multicolumn{2}{|l|}{ Lower jaw } \\
\hline 0 & 33 & \multicolumn{2}{|l|}{50} \\
\hline$<33 \%$ & 22 & \multicolumn{2}{|l|}{40} \\
\hline$<67 \%$ & 31 & \multicolumn{2}{|l|}{47} \\
\hline \multirow[t]{3}{*}{$>67 \%$} & 28 & 40 & \\
\hline & \multicolumn{3}{|c|}{ Implant diameter } \\
\hline & $3.5 \mathrm{~mm}$ & $4.5 \mathrm{~mm}$ & $5.5 \mathrm{~mm}$ \\
\hline 0 & 67 & 14 & 2 \\
\hline$<33 \%$ & 47 & 15 & 0 \\
\hline$<67 \%$ & 63 & 14 & 1 \\
\hline \multirow[t]{3}{*}{$>67 \%$} & 48 & 18 & 2 \\
\hline & \multicolumn{3}{|l|}{ KM } \\
\hline & $<2 \mathrm{~mm}$ & \multicolumn{2}{|l|}{$\geq 2 \mathrm{~mm}$} \\
\hline 0 & 24 & \multicolumn{2}{|l|}{59} \\
\hline$<33 \%$ & 21 & \multicolumn{2}{|l|}{41} \\
\hline$<67 \%$ & 17 & \multicolumn{2}{|l|}{61} \\
\hline$>67 \%$ & 23 & \multicolumn{2}{|l|}{45} \\
\hline \multirow[t]{2}{*}{ (2) PD values } & \multicolumn{3}{|c|}{ Implant Region } \\
\hline & Upper jaw & \multicolumn{2}{|l|}{ Lower jaw } \\
\hline $1-3 \mathrm{~mm}$ & 74 & \multicolumn{2}{|l|}{161} \\
\hline $4-6 \mathrm{~mm}$ & 40 & \multicolumn{2}{|l|}{16} \\
\hline \multirow[t]{3}{*}{$>7 \mathrm{~mm}$} & 0 & \multicolumn{2}{|l|}{0} \\
\hline & \multicolumn{3}{|c|}{ Implant diameter } \\
\hline & $3.5 \mathrm{~mm}$ & $4.5 \mathrm{~mm}$ & $5.5 \mathrm{~mm}$ \\
\hline $1-3 \mathrm{~mm}$ & 188 & 45 & 2 \\
\hline 4-6 mm & 37 & 16 & 3 \\
\hline \multirow[t]{3}{*}{$>7 \mathrm{~mm}$} & 0 & 0 & 0 \\
\hline & \multicolumn{3}{|l|}{ KM } \\
\hline & $<2 \mathrm{~mm}$ & \multicolumn{2}{|l|}{$\geq 2 \mathrm{~mm}$} \\
\hline $1-3 \mathrm{~mm}$ & 70 & 165 & \\
\hline $4-6 \mathrm{~mm}$ & 15 & 41 & \\
\hline$>7 \mathrm{~mm}$ & 0 & 0 & \\
\hline (3) MR & Implant re & & \\
\hline & Upper jaw & Lower jaw & \\
\hline $0 \mathrm{~mm}$ & 72 & 119 & \\
\hline$>0 \mathrm{~mm}$ & 42 & 58 & \\
\hline & Implant di & & \\
\hline & $3.5 \mathrm{~mm}$ & $4.5 \mathrm{~mm}$ & $5.5 \mathrm{~mm}$ \\
\hline $0 \mathrm{~mm}$ & 145 & 42 & 4 \\
\hline$>0 \mathrm{~mm}$ & 80 & 19 & 1 \\
\hline & KM & & \\
\hline & $<2 \mathrm{~mm}$ & $>2 \mathrm{~mm}$ & \\
\hline
\end{tabular}

Table 4 Test group ( $n=291$ implants) cross-tabulations: a) BOP/PD/MR values and (1) implant region, (2) implant diameter, and (3) KM (Continued)

\begin{tabular}{lcc}
\hline $\mathbf{0 ~} \mathbf{~ m m}$ & 43 & 148 \\
$>\mathbf{0 ~} \mathbf{~ m m}$ & 42 & 58 \\
\hline $\begin{array}{l}B O P \text { bleeding on probing, } \\
\text { keratinized mucosa }\end{array}$ &
\end{tabular}

placed in pristine bone without hard tissue grafting. The implant-level analysis, however, pointed toward higher mean PD values for the grafted implant sites (3.0 vs. $2.78 \mathrm{~mm}$ ). Our findings basically corroborate the results of one previous randomized clinical trial that elaborated upon the effects of lateral bone grafting around dental implants presenting with dehiscence-type defects $(\leq 5$ $\mathrm{mm})$ at the vestibular aspect over a spontaneous defect healing (i.e., without grafting) $[8,9]$. In particular, a more pronounced vertical bone loss during the first 6 months following implant placement as well as significantly higher residual vertical dehiscence defects was noted following spontaneous healing, as compared to the grafted implant sites $(-0.17 \mathrm{~mm}$ and 2.51 vs. 1.79 $\mathrm{mm}$ and 1.61 , respectively) $[8,9]$. Nevertheless, the clinical performance of the implants in the test and control groups was similar, and peri-implant tissue health with low mucosal inflammatory (i.e., sulcus bleeding index) changes was maintained over the mean investigation period of 7.5 years [8].

Opposing clinical data, on the other hand, have demonstrated that residual dehiscence-type defects and subsequently exposed rough implant surfaces negatively influenced peri-implant tissue health [10]. In particular, after 4 years, implant sites exhibiting advanced residual dehiscence-type defects $(>1 \mathrm{~mm})$ on the vestibular aspect following simultaneous lateral grafting (natural bone mineral + native or crosslinked collagen membranes) revealed an increased risk of developing peri-implant tissue inflammation, thus emphasizing the need for optimizing defect fill when performing bone grafting simultaneous with implant placement [10]. Further results from the aforementioned investigation showed a tendency toward higher MR values, especially on the midbuccal aspect of implant sites exhibiting residual defects of $>1 \mathrm{~mm}$ [10]. The latter observation is in accordance with the results of a recent cross-sectional analysis, which detected a more apical position of the first bone-toimplant contact at the vestibular aspect for implants presenting with mucosal recessions $(4.85 \mathrm{~mm}$ vs. 2.15 $\mathrm{mm}$, respectively) [11]. Similar findings were also observed at immediately placed implants, since implant sites showing no radiographic bone at the buccal site were associated with an apical shift $(1 \mathrm{~mm})$ of the mucosal level when compared with implants showing 
Table 5 Control group ( $n=283$ implants) cross-tabulations: a) OP/PD/MR values and (1) implant region, (2) implant diameter, and (3) KM

\begin{tabular}{|c|c|c|c|}
\hline \multirow[t]{2}{*}{ 1) BOP values } & \multicolumn{3}{|c|}{ Implant region } \\
\hline & Upper jaw & \multicolumn{2}{|l|}{ Lower jaw } \\
\hline 0 & 59 & \multicolumn{2}{|l|}{20} \\
\hline$<33 \%$ & 33 & \multicolumn{2}{|l|}{13} \\
\hline$<67 \%$ & 56 & \multicolumn{2}{|l|}{26} \\
\hline \multirow[t]{3}{*}{$>67 \%$} & 57 & 19 & \\
\hline & \multicolumn{3}{|c|}{ Implant diameter } \\
\hline & $3.5 \mathrm{~mm}$ & $4.5 \mathrm{~mm}$ & $5.5 \mathrm{~mm}$ \\
\hline 0 & 64 & 15 & 0 \\
\hline$<33 \%$ & 35 & 11 & 0 \\
\hline$<67 \%$ & 69 & 12 & 1 \\
\hline \multirow[t]{3}{*}{$>67 \%$} & 65 & 10 & 1 \\
\hline & \multicolumn{3}{|l|}{ KM } \\
\hline & $<2 \mathrm{~mm}$ & \multicolumn{2}{|l|}{$>2 \mathrm{~mm}$} \\
\hline 0 & 12 & \multicolumn{2}{|l|}{67} \\
\hline$<33 \%$ & 12 & \multicolumn{2}{|l|}{34} \\
\hline$<67 \%$ & 19 & \multicolumn{2}{|l|}{63} \\
\hline$>67 \%$ & 22 & \multicolumn{2}{|l|}{54} \\
\hline \multirow[t]{2}{*}{ (2) PD values } & \multicolumn{3}{|c|}{ Implant region } \\
\hline & Upper jaw & \multicolumn{2}{|l|}{ Lower jaw } \\
\hline $1-3 \mathrm{~mm}$ & 138 & \multicolumn{2}{|l|}{70} \\
\hline $4-6 \mathrm{~mm}$ & 64 & \multicolumn{2}{|l|}{8} \\
\hline \multirow[t]{3}{*}{$>7 \mathrm{~mm}$} & 3 & \multicolumn{2}{|l|}{0} \\
\hline & \multicolumn{3}{|c|}{ Implant diameter } \\
\hline & $3.5 \mathrm{~mm}$ & $4.5 \mathrm{~mm}$ & $5.5 \mathrm{~mm}$ \\
\hline $1-3 \mathrm{~mm}$ & 174 & 33 & 1 \\
\hline 4-6 mm & 57 & 14 & 1 \\
\hline \multirow[t]{3}{*}{$>7 \mathrm{~mm}$} & 2 & 1 & 0 \\
\hline & \multicolumn{3}{|l|}{$\mathrm{KM}$} \\
\hline & $<2 \mathrm{~mm}$ & \multicolumn{2}{|l|}{$>2 \mathrm{~mm}$} \\
\hline 1-3 mm & 41 & 167 & \\
\hline $4-6 \mathrm{~mm}$ & 23 & 49 & \\
\hline$>7 \mathrm{~mm}$ & 1 & 2 & \\
\hline (3) MR & Implant rec & & \\
\hline & Upper jaw & Lower jaw & \\
\hline $0 \mathrm{~mm}$ & 153 & 55 & \\
\hline$>0 \mathrm{~mm}$ & 52 & 23 & \\
\hline & Implant dia & & \\
\hline & $3.5 \mathrm{~mm}$ & $4.5 \mathrm{~mm}$ & $5.5 \mathrm{~mm}$ \\
\hline $0 \mathrm{~mm}$ & 172 & 34 & 2 \\
\hline$>0 \mathrm{~mm}$ & 61 & 14 & 0 \\
\hline & KM & & \\
\hline & $<2 \mathrm{~mm}$ & $>2 \mathrm{~mm}$ & \\
\hline
\end{tabular}

Table 5 Control group ( $n=283$ implants) cross-tabulations: a) OP/PD/MR values and (1) implant region, (2) implant diameter, and (3) KM (Continued)

\begin{tabular}{lll}
\hline $\mathbf{0} \mathbf{~ m m}$ & 29 & 179 \\
$>\mathbf{0 ~} \mathbf{~ m m}$ & 36 & 39 \\
\hline BOP bleeding on probing, & $P D$ probing depth, & MR mucosal recession, $K M$
\end{tabular}
keratinized mucosa

an intact buccal bone [12]. In the present analysis, the overall mean MR values were low and similar at both test and control implant sites $(0.18 \mathrm{~mm}$ and $0.17 \mathrm{~mm}$, respectively). This is in disagreement with the previous data suggesting more favorable mucosal levels at implant sites treated without GBR than at sites that underwent lateral GBR [9]. In this context, it must be emphasized that the buccal bone levels were not evaluated in the present study and therefore, the potential presence of residual defects in the test group could not be assessed. Apart from that, periimplant tissue health and esthetics appeared to not be jeopardized by either thin or missing vestibular bone at the implants in the maxillary anterior region [13]. One might speculate that those findings, to some certain extent, could be explained by the inverse relationship noted between bone and soft-tissue thickness [14]. In particular, one pre-clinical study employing a canine model observed a physiological increase in the peri-implant mucosa thickness as a compensation for underlying vestibular bone deficiencies, with the highest horizontal mucosa thickness detected in the absence of vestibular bone plate [14].

When further interpreting the results of the present study, the regression analysis for the test implant sites revealed a significant association between larger implant diameter (i.e., $4.5-5.5 \mathrm{~mm}$ vs. $3.5 \mathrm{~mm}$ ) and increased PD values (i.e., $4-6 \mathrm{~mm}$ ). This finding might be partially attributable to the fact that inserting a larger implant diameter may lead to an implant location being outside of the bony envelope, resulting in uncontained peri-implant defects. From a biological perspective, such defects feature a compromised regenerative potential; thus, the presence of increased PDs may be a clinical sign pointing to an incomplete defect regeneration.

Regression analysis also demonstrated that control implant sites with reduced $\mathrm{KM}$ values $(<2 \mathrm{~mm})$ were frequently associated with profuse mucosal bleeding ( $67 \%$ ) and increased PD values of $4-6 \mathrm{~mm}$ compared to the implants exhibiting $\mathrm{KM} \geq 2 \mathrm{~mm}$. This tendency may be related to the fact that reduced KM width (< $2 \mathrm{~mm}$ ) was shown to negatively affect self-performed oral hygiene measures, which subsequently increased the likelihood of peri-implant mucosal inflammation [15]. Another noteworthy finding of the present study 
was the increased risk for mucosal recession at implants exhibiting $\mathrm{KM}$ of $<2 \mathrm{~mm}$. This tendency aligns with the results of one previous cross-sectional analysis, which found $\mathrm{KM}$ of $>2 \mathrm{~mm}$ being a protective factor against peri-implant soft-tissue dehiscence, whereas a more apical soft-tissue position on the vestibular aspect was frequently detected at the implant sites with $\mathrm{KM}<2 \mathrm{~mm}$ [11].

The present prevalence of peri-implant mucositis and peri-implantitis between the test and control groups was similar, amounting to $66 \%$ and $5 \%$ in the implant test group and $64 \%$ and $8 \%$ in the control group, respectively. A slightly higher frequency of peri-implant mucositis was indicated in a recent clinical trial, where $81 \%$ of the implants in the test group (i.e., dehiscence defects treated with lateral hard tissue grafting) and $79 \%$ in the control group (i.e., spontaneous defect healing) presented with bleeding upon probing procedure [8]. Note that in contrast to the results of the present study, none of the implants developed signs of periimplantitis over the investigation period of 7.5 years [8]. The existing contradicting data, however, pointed toward a link between bone augmentation procedures and increased peri-implantitis risk $(\mathrm{OR}=2)$ [16]. Moreover, patients exhibiting implants placed along with the bone grafting procedures had more than double the frequency of peri-implantitis (defined as BOP+ and/or suppuration, $\mathrm{PD} \geq 4 \mathrm{~mm}$, radiographic bone level $>3$ ), as compared to those having implants inserted into the pristine bone (18\% vs. $7 \%$ ) [17].

Within the limitations of the present study, it was concluded that simultaneous lateral grafting was associated with peri-implant tissue health and stability.

\section{Acknowledgements}

Not applicable.

\section{Authors' contributions}

AR and FS have made substantial contributions to study the conception and interpretation of data as well as manuscript drafting. $A B, P P$, and MEGV were involved in the data management as well as the statistical analysis. KO contributed to the data interpretation and manuscript drafting. All authors read and approved the final manuscript.

\section{Funding}

The study was self-funded by the authors own departments.

\section{Availability of data and materials}

Not applicable.

\section{Ethics approval and consent to participate}

The study protocol was approved by the Goethe University (Frankfurt, Germany) ethics committee (register number 78/18).

\section{Consent for publication}

Not applicable.

\section{Competing interests}

The authors Ausra Ramanauskaite, Frank Schwarz, Amira Begic, Puria Parvini, Maria Elisa Galarraga-Vinueza, and Karina Obreja stated explicitly that there are no conflicts of interest related to this article.

\section{Author details}

'Department of Oral Surgery and Implantology, Carolinum, Goethe University, Theodor-Stern-Kai 7, Building 29, 60596 Frankfurt am Main, Germany. ${ }^{2}$ Department of Oral Surgery and Implantology, Carolinum, Johann Wolfgang Goethe-University Frankfurt, 60596 Frankfurt am Main, Germany. ${ }^{3}$ Federal University of Santa Catarina (UFSC), Florianópolis, SC, Brazil.

${ }^{4}$ Department of Orthodontics, Westdeutsche Kieferklinik, Universitätsklinikum Düsseldorf, 40225 Düsseldorf, Germany.

Received: 16 March 2020 Accepted: 6 May 2020

Published online: 12 August 2020

\section{References}

1. Hammerle CH, Araujo MG, Simion M. Evidence-based knowledge on the biology and treatment of extraction sockets. Clin Oral Implants Res. 2012; 23(Suppl 5):80-2.

2. Naenni $\mathrm{N}$, et al. Efficacy of lateral bone augmentation prior to implant placement: a systematic review and meta-analysis. J Clin Periodontol. 2019; 46(Suppl 21):287-306.

3. Thoma DS, et al. Efficacy of lateral bone augmentation performed simultaneously with dental implant placement: a systematic review and meta-analysis. J Clin Periodontol. 2019;46(Suppl 21):257-76.

4. Sanz-Sanchez l, et al. Effects of lateral bone augmentation procedures on peri-implant health or disease: a systematic review and meta-analysis. Clin Oral Implants Res. 2018;29(Suppl 15):18-31.

5. Schwarz F, et al. Evidence-based knowledge on the aesthetics and maintenance of peri-implant soft tissues: Osteology Foundation Consensus Report Part 2-Effects of hard tissue augmentation procedures on the maintenance of peri-implant tissues. Clin Oral Implants Res. 2018;29(Suppl 15):11-3.

6. Loe $\mathrm{H}$. The gingival index, the plaque index and the retention index systems. J Periodontol. 1967;38((6):Suppl):610-6.

7. Berglundh T, et al. Peri-implant diseases and conditions: Consensus report of workgroup 4 of the 2017 World Workshop on the Classification of Periodontal and Peri-Implant Diseases and Conditions. J Periodontol. 2018; 89(Suppl 1):S313-s318.

8. Waller T, et al. Long-term clinical and radiographic results after treatment or no treatment of small buccal bone dehiscences at posterior dental implants: a randomized, controlled clinical trial. Clin Oral Implants Res. 2020.

9. Jung RE, et al. A randomized controlled clinical trial comparing small buccal dehiscence defects around dental implants treated with guided bone regeneration or left for spontaneous healing. Clin Oral Implants Res. 2017; 28(3):348-54.

10. Schwarz F, Sahm N, Becker J. Impact of the outcome of guided bone regeneration in dehiscence-type defects on the long-term stability of periimplant health: clinical observations at 4 years. Clin Oral Implants Res. 2012; 23(2):191-6

11. Sanz-Martin I, et al. Factors associated with the presence of peri-implant buccal soft tissue dehiscences. a case-control study. J Periodontol. 2020.

12. Benic Gl, et al. Dimensions of buccal bone and mucosa at immediately placed implants after 7 years: a clinical and cone beam computed tomography study. Clin Oral Implants Res. 2012;23(5):560-6.

13. Veltri $\mathrm{M}$, et al. Three-dimensional buccal bone anatomy and aesthetic outcome of single dental implants replacing maxillary incisors. Clin Oral Implants Res. 2016;27(8):956-63.

14. Schwarz F, et al. Horizontal mucosal thickness at implant sites as it correlates with the integrity and thickness of the buccal bone plate. Clin Oral Implants Res. 2016;27(10):1305-9.

15. Schwarz F, et al. Peri-implantitis. J Periodontol. 2018;89(Suppl 1):S267-s290.

16. Canullo $L$, et al. Distinguishing predictive profiles for patient-based risk assessment and diagnostics of plaque induced, surgically and prosthetically triggered peri-implantitis. Clin Oral Implants Res. 2016;27(10):1243-50.

17. Canullo $L$, et al. Clinical and microbiological findings in patients with periimplantitis: a cross-sectional study. Clin Oral Implants Res. 2016;27(3):376-82.

\section{Publisher's Note}

Springer Nature remains neutral with regard to jurisdictional claims in published maps and institutional affiliations. 